\title{
TURNING NORTON'S DOME AGAINST MATERIAL INDUCTION
}

\author{
Richard Dawid
}

\begin{abstract}
John Norton has proposed a position of "material induction" that denies the existence of a universal inductive inference schema behind scientific reasoning. In this vein, Norton has recently presented a "dome-scenario" based on Newtonian physics that, in his understanding, is at variance with Bayesianism. The present note points out that a closer analysis of the dome-scenario reveals incompatibilities with material inductivism itself.
\end{abstract}

\section{1: Introduction}

In a number of recent papers (2003, 2007, 2008), John Norton has developed a position of "material induction" that denies the existence of a universal inductive inference schema behind scientific reasoning. Norton argues that schemas which have been developed to date in order to provide a systematic foundation for all forms of inductive inference in science always suffer from either of two fatal problems: they are too general to enforce the necessary restrictions on inductive praxis or they fail to fulfil their claim of universality by being inapplicable to some examples of inductive inference in science. Norton draws the conclusion that the hierarchies should be inverted. Rather than understand the principle of induction as a meta-scientific principle that defines the basic character of all allowed inference patterns in science, he suggests viewing inductive inference schemas in science as being exhaustively determined by the scientists' knowledge about the given context of scientific reasoning. All viable instances of inductive inference on that view are licensed by valid scientific theories and/or by other facts regarding the context of scientific research (like the quality or trustworthiness of involved scientists, etc). Each instance of inductive inference may in principle be withdrawn or altered based on the acceptance of a new framework of scientific theories or new information about more general facts. No room thus remains for a universal principle of induction that can be formulated independently from the scientific status quo.

Beyond suggesting a new take on the character of inductive reasoning in science, the material theory of induction also affects the understanding of the Humean problem of induction. While Norton concedes that the principle of material induction does not offer an outright solution to the Humean problem, he claims that, at any rate, it makes the latter much more difficult to formulate: if present day reasoning is fully justifiable based on scientific background knowledge, the elements of genuine Humean induction retreat to the archaic construction periods of human world views, where they are notoriously difficult to grasp.

The currently most prominent conception that proposes one universal schema for inductive reasoning and therefore is rejected by the material theory of induction is Bayesianism. Bayesianism asserts that all scientific reasoning is based on attributing a 
probability to a theory's or a statement's viability. That probability measures the degree of belief a scientist has in the given theory or prediction. Experiments on this account alter a theory's or statement's probability according to the following formula:

$$
P(H / E)=\frac{P(E / H)}{P(E)} P(H)
$$

where $\mathrm{P}(\mathrm{H})$ is the prior probability of the hypothesis $\mathrm{H}$ before the empirical data $\mathrm{E}$ has been considered; $\mathrm{P}(\mathrm{E})$ is the prior probability of the empirical data $\mathrm{E}$ disregarding $\mathrm{H} ; \mathrm{P}(\mathrm{H} / \mathrm{E})$ is the probability for $\mathrm{H}$ when $\mathrm{E}$ has been taken into account; and $\mathrm{P}(\mathrm{E} / \mathrm{H})$ is the probability for $\mathrm{E}$ given that $\mathrm{H}$ is true. Probabilities according to this schema can only be assigned based on prior probabilities attributed to theories and empirical data, which themselves must either be posited or assessed based on another Bayesian procedure. No algorithm exists for determining initial probabilities. According to the Bayesian, this does not constitute a serious problem for Bayesianism because repeated empirical tests tend to create 'fixed points' for probabilities that are derived from a wide range of early prior probabilities. Probabilities in mature science are thus taken to be fairly well 'decoupled' from the probabilities attributed at early stages of the scientific development.

In (2007, 2008), Norton argues against Bayesianism by suggesting that specific forms of inductive reasoning in science do not conform to the schema laid out by Bayesianism. He exemplifies his claim in the context of classical Newtonian physics. The latter constitutes a prime example of a well confirmed scientific theory. To put it in Bayesian terms, we attribute a high probability to the viability of Newtonian physics within certain limits. Newtonian physics thus should be expected to adhere to the Bayesian principles of inductive inference if the latter constitute general characteristics of scientific reasoning. Norton now points out that Newtonian physics allows for various instances of indeterminacy which cannot be grasped based on probabilistic statements and therefore imply inference schemes which are not covered by Bayesianism.

The example he discusses most extensively is a circular symmetric dome with a height

$$
\mathrm{h}=(2 / 3 \mathrm{~g}) \mathrm{r}^{3 / 2},
$$

where $r$ is the radial distance on the surface, and $g$ the gravitational acceleration. ${ }^{1}$ If the system is assumed to be frictionless, the Newtonian equations of motion for a point-like massive 'ball' put at the dome's apex turns out to have solutions for any value of $t$, where $t$ denotes the elapsed time between the moment when the ball is put on top of the dome and the moment when the ball starts moving downwards. ${ }^{2}$ There is also the solution that the ball stays on top forever. In other words, Newtonian physics tells us that the ball can slide down but it leaves undetermined whether and when it actually does so. The theory does not even give a probabilistic prediction with respect to the latter question. It states a possibility without providing any probability for that possibility to be instantiated ${ }^{3}$. Norton argues that a scientist who is confronted with such a situation has to adhere to her scientific formulas and believe in a possibility without assuming a probability for the ball's movement. This, however,

\footnotetext{
${ }^{1}$ For a full presentation of the dome scenario, see (Norton 2003a).

${ }^{2}$ The individual idealisations in the model are of different status. The assumption of point-likeness is introduced in order to construct the simplest possible indeterminist system. Lack of friction is a precondition for having an indeterminate system at all.

${ }^{3}$ Intuitively, the situation can be grasped more easily by looking at the time reversed system. The system then has solutions where a ball pushed upwards stops exactly at the apex after a finite period of time.
} 
contradicts the Bayesian claim that all statements about the world must be inherently probabilistic. $^{4}$

The present note will not discuss Norton's assessment that the dome example contradicts a Bayesian approach. It will make the point, however, that the example actually is at variance with a rigid understanding of material induction. In one crucial respect it will actually turn out to be less capable of accounting for the dome scenario's implications than its Bayesian rival.

\section{2: The Argument against Material Induction}

Before entering the discussion of the dome's implications for material induction, we have to make some qualifications. The dome is an idealized model that does not conform to the world as we know it. It is based on pure Newtonian physics, neglecting friction and quantum effects. Moreover, the initial conditions which lead to the indeterminacy phenomenon have measure zero in the space of all possible initial conditions even when a finite volume is attributed to the overall system. A mechanical process that can only be tuned within some finite range of error thus has a zero chance of producing the initial condition that implies the indeterminacy. The dome example therefore is a scenario without actual empirical relevance. Still, the example can be invoked against principled claims on scientific thinking. The contingent fact that, at the present time, we do not know of scientific conceptions where this specific form of indeterminism is empirically relevant (who knows whether that may change in the future?) does not affect the point that our methodology of scientific reasoning about the world does allow for non-probabilistic claims.

In order to understand the problem faced by material induction in the given context, it is important to distinguish two parts of the dome scenario: a theoretical part that consists in everything Newtonian physics has to say about the dome scenario; and an empirical part that is represented by the notion of an empirical test of the dome system's properties. It is essential to take the empirical part into consideration since only the understanding as to how empirical data can affect a theory's validity makes that theory a genuine element of natural science. In the following, we will thus treat the dome example as a thought experiment that includes both the scientific model and its empirical testing. To that end, we will assume that it is possible for an "idealized experimentalist" to create the initial conditions for indeterminacy.

In (2007, 2008), Norton only addresses the dome scenario's theoretical part. Both, his criticism of Bayesianism as well as his assessment of material induction are discussed with respect to the theoretical part of the dome scenario. Norton can rightly point out that material induction nicely accounts for the dome at that level. Rather than suggesting any universal inference schema, the material inductivist asserts that science fully determines the inference schemas. That position is neatly exemplified by the dome scenario. Applied to the dome, Newtonian physics determines the ball's possible behaviour but does not offer any probabilities with respect to the time a ball spends on the dome's apex before it starts sliding down. The fact that inductive inference in the given case leads to a statement on potentiality without stating a probability is indeed fully determined by the scientific theory itself.

Let us now go beyond Norton's analysis by turning to the scenario's second part, to the empirical testing of the dome-ball system's behaviour. We assume that an idealized experimentalist tests the dome's properties by building a hundred domes, putting balls on top of each of them and measuring the times $t$ when each of these balls start sliding. We have

\footnotetext{
${ }^{4}$ (Malament 2008) points out that a number of reasons to doubt whether Norton's dome is adequately described as an indication that "Newtonian particle mechanics is an indeterministic theory". In the following, we will adhere to the advice given in the paper itself, and "'avoid labels here and direct attention, instead, to a rich set of issues that the example raises".
} 
already noted that the scientific status quo does not attribute prior probabilities to any potential outcome of the sliding ball experiment.

In order to understand the material inductivist position in the given case, we have to look closer at the experiment's possible outcomes. At first glance, it may seem that, in view of what has been said in section one, Newtonian mechanics predicts the absence of any stable regularity pattern or definite shape of distribution with respect to t. It is easy to understand, however, that this is not correct. The system's indeterminacy implies that Newtonian physics cannot be refuted by any empirical data on the times when balls start sliding from the dome's apex. Any imaginable distribution pattern of starting times for sliding is compatible with Newtonian mechanics. If all hundred balls in the experiment above start sliding exactly after 16,8 seconds, this does not contradict the theory any more than any other experimental outcome. It is true, of course, that Newtonian mechanics has nothing to say about the outcome of $101^{\text {st }}$ experiment even after one hundred experiments have been carried out with identical results. That, however, is exactly the point where the dome turns against material induction.

Let us imagine that the scientist doing the dome experiment indeed measures 100 times out of 100 experimental runs $\mathrm{t}=16,8 \mathrm{~s}$. The rigid material inductivist must adhere to the following line of reasoning in that case.

- Inductive inference in science is always and entirely motivated by the scientists' knowledge about the world.

- The description of the dome example relies exclusively on Newtonian physics.

- Newtonian physics remains completely silent with respect to regularity patterns of the times $t$ when the balls start sliding from the top of the dome.

$\Rightarrow$ The empirical data collected on that matter can not be used for inductive reasoning.

$\Rightarrow$ The experimenter who sees a hundred balls start sliding consistently after 16,8 seconds thus is not allowed to make an inference from that observation to the next ball's behaviour.

This, however, is quite obviously no advice a scientist in the given situation would follow. An experimenter in her right mind would rather say something like this: the regularity pattern shown by experiment seems to justify the prediction that the next balls in the same experimental setting will start sliding after 16,8 seconds; our present scientific knowledge is not at variance with this regularity but does not give us the slightest clue as to why it occurs. In other words, it seems that the experimenter would apply a scheme of inductive inference that is not based on her scientific knowledge about the world. ${ }^{5}$

Norton responds to this thought experiment the following way (email communication, Nov. 2009):

When a scientist experimenting on a dome finds this result of motion at 16.8 seconds in one hundred of one hundred trials, the scientist would likely conclude that the background Newtonian theory is either incomplete or incorrect. This inductive inference is warranted by a deeper fact presumed in science, that phenomena as regular as this are law-governed. The complete law cannot be existing Newtonian theory since it does not predict this regularity. Alternatively, the extraordinary nature of the result might lead the scientist to speculate that other more prosaic facts might obtain, such as the employing of a dishonest lab assistant. On the warrant of that fact, the scientist might infer that the anomalous result arose through fraud. All this conforms well with the material theory of induction.

\footnotetext{
${ }^{5}$ The experimenter does, of course, rely on scientific knowledge for framing the question, defining the class of relevant experiments, etc.. There is no scientific knowledge, however, that can support or contradict the inductive inference itself.
} 
In the second part of his response, Norton emphasises that explanations of the regularity pattern in question need not be based on scientific theories but could also rely on external reasoning regarding the experimental process. Resorting to explanations of that kind would be in full agreement with material induction. We fully agree on that point. We want to assume in our example, however, that our scientific observer does not find any plausible explanation of the observed regularity in terms of external influences. We assume that no viable or plausible explanation of the observed regularity pattern, be it external or based on a new scientific theory, is available to the scientist.

Regarding that case, Norton offers the following argument. He suggests that, even if no scientific theory capable of accounting for the observed regularity was available, "metainductive" reasoning would lead the scientist to expect that an as yet unknown theory with that capability existed. Scientists have learned to presume that conspicuous and stable observational regularity patterns are law-governed. Norton goes on to argue that a scientist who applies inductive inference in the given case would in fact base that step on assuming the existence of as yet unknown natural laws governing the observed regularity. He then claims that the latter assumption is sufficient for vindicating the principle of material induction.

We share Norton's understanding that scientists would expect the existence of an unknown theoretical scheme if they were confronted with the regularity pattern of our example. ${ }^{6}$ However, we disagree with Norton's claim that this expectation is of significance for an understanding of inductive inference in the given case.

The step of inductive inference we are interested in leads from acknowledging the regularity pattern in the collected data towards predicting that the next events measured in the same empirical setting will be in agreement with that regularity pattern. Let us imagine that the scientist who analyses the data assumes the existence of some unknown theoretical reason for the observed regularity without being able to specify the corresponding theory. The unknown theory she is assuming then might in principle predict any imaginable outcome for future events. The mere assumption that an unspecified theory explains the observed regularity pattern offers no basis for any kind of inductive inference. In order to base inductive inference on the assumption of a theoretical background, the scientist must deliberately exclude those potential theories which make predictions that are at variance with the straightforward extrapolation of the observed regularity pattern. In order to legitimize that step, however, she would have to rely on a corresponding inductive presumption already. The resulting commitment to a restricted class of theoretical conceptions thus could not serve as a basis for inductive reasoning itself. To conclude, there is only one way to carry out inductive inference in the given case: resorting to raw enumerative induction.

Admittedly, the scientist thereby finds herself in a rather unusual and uncomfortable situation. She has no scientific basis whatsoever for determining the probability that a conjecture concerning a regularity pattern for $t$ be viable. She has no basis for defining a natural measure on the space of possible t's, no basis for formulating a null-hypothesis, and no cogent basis for defining a natural ensemble of equivalent experimental runs (i.e. no basis for deciding whether $t$ should depend on the position of the dome, its size, absolute time, etc.). Scientific enquiry in the given case would have to rely on initial conjectures based on raw enumerative induction - a form of induction that lacks a probabilistic basis for testing the corresponding conjecture.

Nevertheless, it would be plainly irrational to ignore obvious and strong regularity patterns. A scientist would have no choice but to develop primitive theories that account for the observed pattern - and have some degree of trust in them if they turn out to be predictive without a solid scientific foundation. Only in a second step, more far-reaching theories could

\footnotetext{
${ }^{6}$ One might add that this expectation could lose strength if no adequate theoretical description was found for a long period of time and if other regularities without general natural laws were observed.
} 
be tested by using a probability calculus based on the assumption that the first conjectures are valid.

Thus, the scientist is forced to do something the material inductivist cannot accept as scientific reasoning: she must interpret regularity patterns discernable in the data based solely on simple unsupported induction without any reference to the web of scientific theories. The dome example therefore demonstrates, contra material induction, that there exists a principle of induction that lies beyond the scientific theories or knowledge of external facts that can be applied in a given scientific context.

\section{3: General Assessment}

The dome-scenario constitutes a more forceful counter-example to material induction than other scientific scenarios that may be understood to be at variance with the latter. It is true, of course, that one can think of many instances in science where observed regularities were neither directly predicted by nor in direct contradiction with the scientific theories known at the time. Any such instance may be taken to support the suspicion that material inductivism is insufficient for giving a universal characterisation of inductive inference. In most of those cases, however, reference to a wider framework of scientific background knowledge offered a basis for relating the observed regularities to the general scientific picture and thereby for assessing their plausibility. For example, the viability of some scientific theories may be extendable to a new regime or general scientific principles may be deployed in a new context to explain a certain experimental outcome. The material inductivist in such cases has the chance to defend his position by claiming that the scientific background knowledge involved fully determines the inductive inferential scheme.

In the dome example, Newtonian physics provides a well defined and universal framework that implies a clear-cut distinction between the deterministic regime, within which most of the phenomena to which the theory is applicable are described, and the indeterministic regime, where the theory explicitly states it has nothing to say. ${ }^{7}$ Thus, the dome provides a "laboratory case" of a situation where the scientific theory itself shields a certain regime from background knowledge by stating explicitly that any regularity pattern found there would be fully coherent with but entirely inexplicable by the scientific status quo. All escape routes for the material inductivist are thus blocked.

The occurrence of raw induction in its pure form in the dome example clearly weakens the general case for material induction. If raw induction can be clearly identified in some scientific contexts, there is no good reason for denying that it occurs elsewhere in science as well. The dome example thus may be taken to show in its pure and isolated form an elementary kind of inductive inference that constitutes a significant though elusive element in many contexts of scientific reasoning.

\footnotetext{
${ }^{7}$ Of course, even the system with a ball at the dome's apex is heavily constrained by physics. We know that the ball will not fly away, not stop on the way down, etc. The indeterministic regime merely comprises one single parameter: $t$, denoting the time when the ball starts sliding.
} 


\section{References}

Howson, C. and Urbach, P. 2006: Scientific Reasoning - The Bayesian Approach, Open Court.

Malament, D. 2008: “Norton’s Slippery Slope”, Philosophy of Science 75/5, 799-816.

Norton, J. 2003: ‘A Material Theory of Induction, Philosophy of Science 70, 647-670.

Norton, J. 2003a: 'Causation as Folk Science' in H.Price and R. Corry (eds), Causation, Physics and the Constitution of Reality', Oxford University Press, 2007.

Norton, J. 2007: 'Probability Disassembled', British Journal of Philosophy of Science 58, 141-171.

Norton, J. 2008: 'There Are No Universal Rules of Induction', held at the PSA 2008, PhilSci. Archive 4273. 